\title{
AS DIFERENTES ABORDAGENS DOS TRABALHOS DE CONCLUSÃO DE CURSO DE GRADUAÇÃO EM ENFERMAGEM DA UNIVERSIDADE FEDERAL DO PARANÁ
}

\author{
DIFFERENT APROACHES ON NURSING GRADUATION CONCLUSION DISSERTATIONS AT THE FEDERAL \\ UNIVERSITY OF PARANÁ
}

DIVERSIDAD DE ABORDAGES EN LAS MONOGRAFIAS DE CONCLUSIÓN DEL CURSO DE GRADUACIÓN EN ENFERMERÍA DE LA UNIVERSIDAD FEDERAL DE PARANÁ

\author{
Maria de Fátima Mantovani ${ }^{1}$ \\ Liliana Maria Labronici ${ }^{2}$ \\ Thais Adriane Leão ${ }^{3}$ \\ Ana Carolina Souza Ribeiro ${ }^{3}$
}

RESUMO: Trata-se da análise retrospectiva dos trabalhos de conclusão elaborados nos últimos 11 anos pelos alunos do Curso de Enfermagem da Universidade Federal do Paraná, que se encontram no acervo da Biblioteca do Setor de Ciências da Saúde. O objetivo do estudo foi identificar as áreas e do tipo de pesquisa realizada pelos acadêmicos. Foram resgatados 240 trabalhos, dos quais $14,6 \%$ na área de saúde da mulher, $7,6 \%$ na área de saúde da criança, $9,7 \%$ na saúde do adulto e do idoso, 3,4\% em fundamentos de Enfermagem, 5,5\% na administração de Enfermagem e saúde e $59,2 \%$ na assistência de Enfermagem em saúde coletiva. Os tipos de estudo foram $81,8 \%$ qualitativos e $18,2 \%$ quantitativos. A partir do ano de 1993 , os relatórios de estágio foram transformados em trabalhos de pesquisa, coincidindo com o esforço dos docentes do curso para a qualificação em nível de mestrado e doutorado.

PALAVRAS - CHAVES: Pesquisa em Enfermagem; Técnicas de Investigação; Conhecimento.

ABSTRACT: It is a retrospect of the graduation monographs elaborated by students of the nursing course at Federal University of Parana along the past eleven years. Such monographs can be found in the Library of Health Sciences. The study objectified the identification of areas and the kind of research undertaken by the graduates. 240 monographs were reviewed, among them $14.6 \%$ were developed in the women's health field, $7.6 \%$ in the child's health field, $9.7 \%$ in the adults'and elders'health field, $3.4 \%$ in Nursing Foundations, $5.5 \%$ in Nursing Management and Health, and $59.2 \%$ in Nursing Practice and Public Health. The kinds of study were $81.8 \%$ qualitative and $18.2 \%$ quantitative. Since 1993, training reports have been changed to research papers, keeping up with the course teachers' efforts to qualify for master's and doctorate's degrees.

KEYWORDS: Nursing Research; Investigative Techniques; Knowledge.

RESUMEN: Se trata de análisis retrospectivo de los trabajos de conclusión elaborados en los ultimos once años, por los alunnos del curso de Enfermaría de la Universidad Federal de Paraná, que se encuentram en la colección de la biblioteca del Sector de Ciencias de la Salud. El objectivo de este estudio fue la identificación de las asreas y del tipo de pesquisa realizada por los académicos. Fueran rescatados 240 trabajos de los cuales el $14,6 \%$ en la area de sanidad de la mujer, el $7,6 \%$ en area de sanidad de los niños, el 9,7\% en el area de sanidad del adulto u del mayor, el $3,4 \%$ en fundamentos de enfermará y salud coletiva. Los tipos de estudios fueran $81,8 \%$ cualitativos y $18,2 \%$ cuantitativos. Desde el año 19993, los informes de las pasantías fueran transformados en trabajos e pesquisa, coincidiendo con el esfuerzo di los docentes del curso para la codificación en el nivel de maestria y dotoracto.

\footnotetext{
${ }^{1}$ Professor Doutor em Enfermagem. Adjunto do Departamento de Enfermagem da Universidade Federal do Paraná. Coordenadora do Gemsa. e-mail: fatmant@ufpr.br. End.: Rua Padre Camargo, $2808^{\circ}$ andar Alto da Glória/Curitiba cep 80060-150

${ }^{2}$ Professor Doutor em Enfermagem. Adjunto do Departamento de Enfermagem da Universidade Federal do Paraná. Membro do Gemsa. email: llabronici@ufpr.br. End.: Rua Padre Camargo, $2808^{\circ}$ andar Alto da Glória/Curitiba cep. 80060-150

${ }^{3}$ Aluna de graduação do curso de Enfermagem da Universidade Federal do Paraná. e-mail: thawag@bol.com.br, caufpr@yahoo.com.br
} 
PALABRAS CLAVES: Investigación em Enfermería; Técnicas Investigagivas; Conocimiento.

\section{INTRODUÇÃO}

A preocupação em ultrapassar a fase técnica para a fase científica na década de 1950, levou a Enfermagem a voltar-se para a organização dos "princípios científicos" da prática de Enfermagem que nesse período era mais intuitiva e baseada na experiência (CARVALHO et al, 2001). Visando atender essa necessidade, é que são estabelecidas, em 1962, as leis que regem o currículo mínimo de Enfermagem, passando o curso para o nível de Ensino Superior.

$\mathrm{Na}$ Universidade Federal do Paraná (UFPR), a proposta de criação do curso de graduação em Enfermagem foi apresentada ao Conselho Setorial do Setor de Saúde da UFPR em 1973, sendo formada uma comissão responsável pela elaboração do seu plano estrutural. Em 1974, o Conselho de Ensino e Pesquisa autoriza a implantação do curso de Enfermagem no Setor de Ciências da Saúde, e em 1975 ocorre o primeiro vestibular, ofertando 40 vagas, sendo 24 para o primeiro semestre e 16 para o segundo.

O reconhecimento do curso pelo Ministério da Educação e Cultura ocorre em 1980, através da portaria $n^{\circ} 100$, e em 1983 além da inserção da habilitação em licenciatura, é ofertado o primeiro curso de especialização Médico- Cirúrgica pelo Departamento de Enfermagem. Desde então, tem ocorrido diversas alterações curriculares, sempre visando melhorar a qualificação do futuro profissional, do ensino e da instituição. Uma das alterações mais significativas para o nosso estudo, refere-se ao estágio supervisionado, inicialmente apresentado como disciplina, que passa a ter sua carga horária incorporada às disciplinas ofertadas.

O estágio supervisionado ofertado no último ano do curso exigia, para fins de avaliação, a elaboração de um relatório das atividades no período, descrevendo o campo, as atividades desenvolvidas e as dificuldades encontradas.

$\mathrm{Na}$ reforma de 1992 é estabelecida a reintrodução da forma de estágio supervisionado como disciplina, e ao invés do relatório das atividades, pediase a elaboração de um trabalho de conclusão de curso na disciplina Enfermagem na Assistência à Saúde. Essa alteração teve como objetivo, estimular o aluno no desenvolvimento de pesquisas e introduzi-lo em novas áreas de atuação, além de promover transformações nas formas de ver e compreender a realidade, transformando o próprio conhecimento, e conseqüentemente, alterar as formas de intervir na realidade (MARTINS, 1994).

Todas essas modificações curriculares propiciaram a produção de trabalhos que até os dias atuais não foram analisados no que diz respeito ao tipo de estudo e adequação dos mesmos ao perfil proposto no projeto pedagógico do curso que é: "formar um profissional que, conhecendo a realidade social na qual esta inserida (o), atua a partir de uma visão crítica sobre a realidade e compromete-se com sua transformação. Deve possuir competência técnicocientifico e política para intervir nos determinantes do processo saúde-doença, respeitando os princípios éticos e legais da profissão" (UFPR, 2002).

A inquietação sobre que linhas de pesquisa ou áreas de conhecimento foram privilegiadas pelos alunos nos incitou a elaborar este estudo que tem como objetivo: identificar as áreas de conhecimento e tipos de pesquisas realizadas pelos alunos do curso de graduação de Enfermagem da UFPR, desde a implantação do trabalho de conclusão do curso.

\section{Caminho percorrido}

Trata-se de um estudo descritivo, de análise dos trabalhos de conclusão do curso de Enfermagem da Universidade Federal do Paraná, classificando-as nas áreas de atuação da Enfermagem e tipo de estudo: qualitativo ou quantitativo.

As áreas utilizadas para classificação foram: Saúde da Mulher, Saúde da Criança, Saúde do Adulto e do Idoso, Fundamentos de Enfermagem, Administração de Enfermagem e Saúde, e Assistência de Enfermagem em Saúde Coletiva.

Foram resgatados 240 trabalhos de conclusão de curso, que se encontram arquivados na Biblioteca do Setor de Saúde da Universidade Federal do Paraná, do período de 1992 a 2002/1.

O estudo foi realizado mediante pedido de autorização aos professores do Departamento de Enfermagem e à responsável pela Biblioteca do Setor de Ciências de Saúde da UFPR.

Os trabalhos de conclusão de curso tiveram seus dados agrupados dentro das áreas de atuação de Enfermagem citadas acima, com os números percentuais. O mesmo foi feito com a descrição do tipo de pesquisa, qualitativo ou quantitativo.

\section{APRESENTAÇÃO E DISCUSSÃO DOS RESULTADOS}

A análise dos 240 trabalhos arquivados na Biblioteca possibilitou a classificação dos mesmos e apresentação dos dados através de gráfico e quadro, contendo informações categorizadas à partir do levantamento dos resultados das áreas de estudo mais abordadas e a classificação da abordagem do tema nas formas qualitativa ou quantitativa.

O estudo dos trabalhos de conclusão de curso resultou nos seguintes dados: 35 na área de Saúde da Mulher, 18 Saúde da Criança, 23 Saúde do Adulto e do Idoso, 8 Fundamentos de Enfermagem, 13 Administração de Enfermagem e Saúde, e 143 Assistência de Enfermagem em Saúde Coletiva. 


\begin{tabular}{|l|l|l|l|l|l|l|}
\hline $\begin{array}{l}\text { Área de } \\
\text { interesse }\end{array}$ & $\begin{array}{l}\text { Saúde } \\
\text { da } \\
\text { Mulher }\end{array}$ & $\begin{array}{l}\text { Saúde da } \\
\text { Criança }\end{array}$ & $\begin{array}{l}\text { Saúde } \\
\text { Coletiva }\end{array}$ & $\begin{array}{l}\text { Saúde do } \\
\text { Adulto e do } \\
\text { Idoso }\end{array}$ & $\begin{array}{l}\text { Fundamentos } \\
\text { doministração }\end{array}$ & Admo \\
\hline Porcentagem & $14,6 \%$ & $7,6 \%$ & $59,2 \%$ & $9,7 \%$ & $3,4 \%$ & $5,5 \%$ \\
\hline
\end{tabular}

Conforme o quadro acima, a área de Assistência de Enfermagem em Saúde Coletiva foi a que concentrou maior percentual de produção, seguida da saúde da mulher, Saúde do Adulto e do Idoso, Saúde da Criança, Administração, e Fundamentos, respectivamente.

\title{
Gráfico I - Tipos de Estudo
}

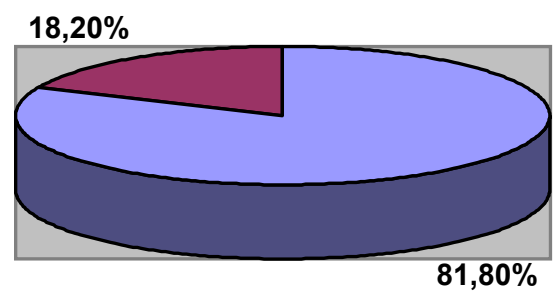

\author{
$\square$ Qualitativo \\ $\square$ Quantitativo
}

Observou-se ainda, quanto ao tipo de pesquisa realizada, que os estudos qualitativos e quantitativos ocorrem na prevalência de $81,8 \%$ e $18,2 \%$, respectivamente. Comparando estes dados com as linhas de pesquisa do Programa de Pós - Graduação em Enfermagem da UFPR: Fundamentos teóricosfilosóficos do cuidar em saúde e Enfermagem; Políticas e praticas de saúde educação e Enfermagem ; Processo de cuidar em saúde e Enfermagem ; Saúde e qualidade de vida podemos observar que estas direcionam os trabalhos para abordagens qualitativas de pesquisa.

\section{Considerações Finais}

A produção do conhecimento da Enfermagem no Brasil vem sendo amplamente estudada e discutida pela comunidade científica da área, sempre buscando identificar os temas mais abordados, as áreas de pesquisa e sobretudo, as falhas/necessidades do processo educativo procedente (ALVES,1998; SAUPE \& GEIB, 2001; SAUPE \& CESTARI, 2002)

No presente estudo, um dos aspectos analisados refere-se à direcionalidade temática, organizadas em áreas e linhas de pesquisa que refletem a abrangências do corpo de conhecimento que vem sendo constituído ao longo do tempo(POLLIT \& HUNGLER, 1992).

Provavelmente o desenvolvimento dos estudos, com destaque para a área de Saúde Coletiva, ocorreu por estarem de acordo com o perfil proposta para os egressos do curso.Em relação ao tipo de estudo, temos a prevalência da pesquisa do tipo qualitativa, que procura entender o fenômeno sob o ponto de vista "holístico", e como processo natural.

\section{REFERÊNCIAS BIBLIOGRÁFICAS}

ALVES, B. Produção/ Reprodução do conhecimento no trabalho na Enfermagem: o conhecimento como forma de estar no mundo. In: GARCIA, T. R.; PAGLIUCA, L. M. F.(Orgs). A construção do conhecimento em enfermagem: coletânea de trabalhos. Fortaleza: RENE, 1998. p. 93 - 144.

CARVALHO, I. M. P.; MELO, R. L.; ANDRAUS, L. M. S. Produção científica de enfermagem em nefrologia, no Brasil, no período de 1989 até 1999. Revista Eletrônica de Enfermage, v.3, n.2, 2001. Disponível em:

http://www.fen.ufg.br/revista/revista3 2/nefron.html. MARTINS, G. A.. Caderno de Pesquisas em Administração. v.0, n.0, p.1-6. 1994. Disponível em: http://www.ead.fea.usp.br/cad-pesq/arquivos/C00art01.pdf.

UFPR. Departamento de Enfermagem. Projeto Político Pedagógico do Curso de Enfermagem. 2002.

POLLIT, D. F.; HUNGLER, B. P. Fundamentos de Pesquisa em Enfermagem. Artes Médicas. $3^{\mathrm{a}}$ edição. 1992.

SAUPE, R.; GEIB; L. T. C. O processo de construção dos projetos politico pedagógicos na enfermagem. 
Revista Eletrônica de Enfermagem, v.3, n.2, jul-dez.

2001.

Disponível

em:

http://www.fen.ufg.br/revista/revista3 2/pppenf.html.

SAUPE, R.; CESTARI, M. E. O trabalho coletivo na construção do projeto político pedagógico dos cursos de enfermagem. Revista Eletrônica de Enfermagem, v.4, n.2, 2002. Disponível em: http://www.fen.ufg.br/revista/revista4 2/coletivo.html.

Texto original recebido em 16/09/2004.

Publicação aprovada em 10/12/2004 